\title{
ON THE EXTENSION OF BOREL MEASURES
}

\author{
S. K. BERBERIAN
}

The class of Borel sets in a locally compact space is the $\sigma$-ring generated by the compact sets [3, p. 219], while the class of weakly Borel sets is the $\sigma$-algebra generated by the closed sets [2]. The object of this note is to show that any measure defined on the class of Borel sets may be extended to the class of weakly Borel sets in a simple and canonical way (Theorem 1). There is a useful application to regular Borel measures (Theorem 2) and, in particular, to Haar measure (Theorem 3).

The first part of our discussion is valid for an arbitrary measure space $(X, \delta, \mu)$ in the sense of Halmos [3, p. 73]. Recall that the sets $E$ in $S$ are called measurable. We say that a subset $A$ of $X$ is locally measurable (with respect to $\delta$ ) if its intersection with every measurable set is measurable, that is, if $E \cap A \in \mathcal{S}$ for every $E$ in $\delta$. We write $\delta_{\lambda}$ for the class of all locally measurable sets; it is easy to see that $\delta_{\lambda}$ is a $\sigma$-algebra containing $\delta$. Moreover, if $\delta_{\lambda}$ is regarded as a ring in the usual way, then $\delta$ is an ideal in $S_{\lambda}$. The measure $\mu$ may be extended to a measure $\mu_{\lambda}$ on $\delta_{\lambda}$ in the following way. For each measurable set $E$, let $\mu^{E}$ be the measure on $S_{\lambda}$ defined by the formula $\mu^{E}(A)$ $=\mu(E \cap A)$; the family of measures $\left\{\mu^{E}: E \in \delta\right\}$ is increasingly directed in the obvious sense, and, defining

$$
\mu_{\lambda}=\operatorname{LUB}\left\{\mu^{E}: E \in \delta\right\},
$$

we obtain a measure on $\delta_{\lambda}[1$, Lemma 11]. Thus,

$$
\mu_{\lambda}(A)=\operatorname{LUB}\{\mu(E \cap A): E \in s\}
$$

for every locally measurable set $A$. Since $S$ is an ideal in $\delta_{\lambda}$, it is easy to see that

$$
\mu_{\lambda}(A)=\operatorname{LUB}\{\mu(E): E \subset A, E \in \delta\}
$$

for every locally measurable set $A$, and it follows that $\mu_{\lambda}$ is an extension of $\mu$. In particular, we have "extended" the measure space $(X, \delta, \mu)$ to a measure space $\left(X, \delta_{\lambda}, \mu_{\lambda}\right)$, where $\delta_{\lambda}$ is a $\sigma$-algebra containing $S$. [We call attention to the following easily verified facts: (i) if $\mu$ is finite (and therefore bounded), then $\mu_{\lambda}$ is also finite; (ii) if $\mu$ is $\sigma$-finite, it does not follow that $\mu_{\lambda}$ is $\sigma$-finite; (iii) if $\delta$ is a $\sigma$-algebra to begin with, then $S_{\lambda}=S$ and the extension collapses.]

Received by the editors December 18, 1963. 
For the rest of the paper, let $X$ be a locally compact space, $B$ the class of Borel sets in $X$, and $B_{w}$ the class of weakly Borel sets. As shown in [2], $B$ is an ideal in $B_{w}$; that is, if $E$ is a Borel set and $A$ is a weakly Borel set, then $E \cap A$ is a Borel set. In other words, $B_{w} \subset B_{\lambda}$. If $\mu$ is any measure defined on $\cap$, let us write $\mu_{\mathrm{w}}$ for the restriction of $\mu_{\lambda}$ to $B_{\mathrm{w}}$; then $\mu_{\mathrm{w}}$ is a measure on $\Theta_{\mathrm{w}}$ which extends $\mu$, and, in view of formula (1), we have the following result:

THEOREM 1. If $\mu$ is any measure defined on the class of Borel sets in a locally compact space $X$, then the formula

$$
\mu_{\mathrm{w}}(A)=\operatorname{LUB}\{\mu(E): E \subset A, E \text { Borel }\}
$$

defines an extension of $\mu$ to a measure on the class of weakly Borel sets. If, moreover, $T$ is a homeomorphism of $X$, and $c$ is a positive real number such that $\mu(T(E))=c \mu(E)$ for all Borel sets $E$, then also

$$
\mu_{\mathrm{w}}(T(A))=c \mu_{\mathrm{w}}(A)
$$

for all weakly Borel sets $A$.

Recall that a Borel measure on $X$ is a measure $\mu$, defined on $B$, such that $\mu(C)<\infty$ for every compact set $C[3$, p. 223]. A Borel measure $\mu$ is regular in case

$$
\mu(E)=\operatorname{LUB}\{\mu(C): C \subset E, \quad C \text { compact }\}
$$

for every Borel set $E[3,52 . F]$. A measure $\rho$ defined on $B_{\mathrm{w}}$ will be called a weakly Borel measure if it is finite for compact sets, in other words, if its restriction to $B$ is a Borel measure. A weakly Borel measure $\rho$ will be called regular in case

$$
\rho(A)=\operatorname{LUB}\{\rho(C): C \subset A, \quad C \text { compact }\}
$$

for every weakly Borel set $A$ (more precisely, $\rho$ is "inner regular" [3, p. 224]). [Incidentally, if $\rho$ is a regular weakly Borel measure, then its restriction to the class of Borel sets is a regular Borel measure $\mu$. The converse is false; indeed, any measure $\mu$ defined on $B$ can be extended to a measure $\rho$ on $\widehat{B}_{\mathrm{w}}$ simply by defining $\rho(A)=\infty$ for all weakly Borel sets $A$ which are not Borel sets.]

THEOREM 2. If $\mu$ is a regular Borel measure on a locally compact space $X$, there exists one and only one regular weakly Borel measure $\rho$ on $X$ which extends $\mu$, namely $\rho=\mu_{\mathrm{w}}$.

Proof. The measure $\rho=\mu_{\mathrm{w}}$ is a weakly Borel extension of $\mu$ by Theorem 1, and the regularity of $\rho$ is immediate from the formulas 
(2) and (4). Uniqueness follows from the fact that a regular weakly Borel measure is determined by its values for compact sets.

The application of the foregoing results to Haar measure is as follows (cf. [4, Theorem 9]):

THEOREM 3. If $X$ is a locally compact topological group, there exists a regular weakly Borel measure $\rho$ on $X$, not identically zero, such that

$$
\rho(s A)=\rho(A)
$$

for every weakly Borel set $A$, and every $s$ in $X$. Such a measure is uniquely determined up to a factor of proportionality. For each $s$ in $X$, there is a positive real number $c(s)$ such that

$$
\rho(A s)=c(s) \rho(A)
$$

for every weakly Borel set $A$.

Proof. Let $\mu$ be any nonzero regular Borel measure on $X$ such that $\mu(s E)=\mu(E)$ for all Borel sets $E$ and all $s$ in $X[3,58 . \mathrm{B}]$. By Theorem 2 , the measure $\rho=\mu_{\mathrm{w}}$ is a regular weakly Borel measure extending $\mu$, and the property (6) results from the last assertion in Theorem 1.

Suppose $\rho^{\prime}$ is any other nonzero, regular, weakly Borel measure on $X$, such that $\rho^{\prime}(s A)=\rho^{\prime}(A)$ identically. The restriction of $\rho^{\prime}$ to the class of Borel sets is evidently a nonzero regular Borel measure $\mu^{\prime}$ such that $\mu^{\prime}(s E)=\mu^{\prime}(E)$ identically. Let $c$ be a positive real number such that $\mu^{\prime}(E)=c \mu(E)$ for all Borel sets $E[3,60 . C]$; in particular, $\rho^{\prime}(C)$ $=c \rho(C)$ for all compact sets $C$, and so $\rho^{\prime}=c \rho$ by regularity.

Finally, if $c(s)$ is the positive real number, depending on $s$, such that $\mu(E s)=c(s) \mu(E)$ for all Borel sets $E[3$, Exercise 60.5], then (7) follows from Theorem 1. Incidentally, the proof of (7) could also be based on the essential uniqueness of $\rho$.

It is a remarkable fact that a nonzero Borel measure $\mu$ on a locally compact group $X$ is uniquely determined by the condition $\mu(s E)$ $=\mu(E)$, up to a factor of proportionality $[3,64 . \mathrm{I}]$, and, in particular, $\mu$ is necessarily regular. We remark that the following measure $\mu_{1}$ defined on $\beta$ is also left invariant: $\mu_{1}(\varnothing)=0, \mu_{1}(E)=\infty$ for all nonempty Borel sets $E$; of course $\mu_{1}$ is not a Borel measure.

Similarly, the property (6) alone does not determine a measure up to proportionality on the class of weakly Borel sets. For example there is the measure $\rho_{1}$ such that $\rho_{1}(\varnothing)=0$ and $\rho_{1}(A)=\infty$ for all nonempty weakly Borel sets $A ; \rho_{1}$ is not a weakly Borel measure. Another example is obtained as follows. Assume that $B \neq B_{w}$, in other words, that $X$ is not $\sigma$-compact. Let $\mu$ be a nonzero (regular) Borel measure on $X$ such that $\mu(s E)=\mu(E)$ identically, and extend $\mu$ to a measure $\rho_{2}$ on 
$\bigotimes_{\mathrm{w}}$ by defining $\rho_{2}(A)=\infty$ for every weakly Borel set $A$ which is not a Borel set; then $\rho_{2}$ is a weakly Borel measure, but may not be regular (see the example below). Still another example is the weakly Borel measure $\rho_{3}$ such that $\rho_{3}(E)=0$ for all Borel sets $E$, and $\rho_{3}(A)=\infty$ for all weakly Borel sets $A$ which are not Borel sets.

For an example of $\rho_{2}$ which is not regular, consider the Cartesian product $X=Y \times Z$, where $Y$ is any infinite compact group, and $Z$ is any uncountable discrete group. Identifying $Y$ with $Y \times\{e\}$, where $e$ is the neutral element of $Z$, we may regard $Y$ as a compact open normal subgroup of the locally compact group $X$, and $X / Y$ is uncountable. Since $Y$ is an infinite compact set in $X, X$ is not discrete. Let $A$ be a subset of $X$ which intersects each coset of $Y$ in exactly one point (axiom of choice); it is easy to see that $A$ is a closed subset of $X$, and so is a weakly Borel set. The cosets of $Y$ are an open covering of $X$, and so every compact set in $X$ is contained in the union of finitely many cosets of $Y$; since $A$ is uncountable, it follows easily that $A$ is not $\sigma$-bounded, and so $A$ is not a Borel set [2]. It also follows that a subset of $A$ is compact if and only if it is finite. Let $\mu$ be a nonzero (regular) Borel measure on $X$ such that $\mu(s E)=\mu(E)$ identically. Since $X$ is not discrete, every finite subset of $X$ has measure zero, and so $\mu_{\mathrm{w}}(A)=0$. But $\rho_{2}(A)=\infty$ by the definition of $\rho_{2}$.

ConCLUDING REMARKs. In the theory of abstract measure spaces, $\sigma$-finiteness is often a key hypothesis; every Borel measure is $\sigma$ finite. In topological spaces, open sets are in the center of the stage; every weakly Borel measure includes all open sets in its domain of definition. The Borel and weakly Borel notions coalesce only for spaces which are $\sigma$-compact. Thus, Borel measures fit in better with abstract measure theory, while weakly Borel measures fit in better with topology. Both the Borel and the weakly Borel points of view are valuable, and it is desirable to be able to pass easily from one to the other; the extension procedure described in Theorem 1 is one possible device for doing this.

\section{REFERENCES}

1. S. K. Berberian, The product of two measures, Amer. Math. Monthly 69 (1962), 961-968.

2. S. K. Berberian and J. F. Jakobsen, A note on Borel sets, Amer. Math. Monthly 70 (1963), 55.

3. P. R. Halmos, Measure theory, Van Nostrand, New York, 1950.

4. E. Hewitt, Integration on locally compact spaces. I, University of Washington Publications in Mathematics 3 (1952), 71-75.

The State University of Iowa 